\title{
Dewetting of Glassy Polymer Films
}

\author{
Vijay Shenoy ${ }^{1 *}$ and Ashutosh Sharma ${ }^{2 \dagger}$ \\ Departments of ${ }^{1}$ Mechanical and ${ }^{2}$ Chemical Engineering \\ Indian Institute of Technology Kanpur, UP 208 016, India
}

(Dated: October 31, 2018)

\begin{abstract}
Dynamics and morphology of hole growth in a film of power hardening viscoplastic solid (yield stress $\sim$ [strain-rate $]^{n}$ ) is investigated. At short-times the growth is exponential and depends on the initial hole size. At long-times, for $n>\frac{1}{3}$, the growth is exponential with a different exponent. However, for $n<\frac{1}{3}$, the hole growth slows; the hole radius approaches an asymptotic value as $t \rightarrow \infty$. The rim shape is highly asymmetric, the height of which has a power law dependence on the hole radius (exponent close to unity for $0.25<n<0.4$ ). The above results explain recent intriguing experiments of Reiter, Phys. Rev. Lett., 87, 186101 (2001).
\end{abstract}

PACS numbers: 68.60-p, 61.41.+e, 68.55.-a, 83.50.-v

Dewetting of largely glassy polymer films below or near their glass transition temperature is a highly intriguing phenomenon [i] in that the usual mechanisms of shape change such as viscous fluid flow, surface diffusion, evaporation/condensation are not dominant [1]. Thus, near glass transition temperature, solid-like plastic yielding and resulting flow of the glassy polymer appears to be the only mechanism of dissipation during dewetting.

Indeed, Reiter [1] recently observed the growth of holes in glassy polymer films with features very unlike those predicted/observed in purely viscous 2, 38 films. The key features of the experiments are (i) the radius of the hole increases with time in an exponential manner (short time behaviour) (ii) the growth slows down considerably at long times, to the extent even of nearly stopping the hole grows with a highly unsymmetric raised rim (iv) the height of the rim is nearly linearly dependent on the radius of the hole.

In this Letter, we investigate the role of plastic yielding and resulting flow on the dynamics and morphology of growing holes in glassy polymer films. Our model is based on two physical premises: (i) the dewetting hole growth is driven by capillary forces, and (ii) the polymeric film, near its glass transition temperature, behaves as a strainrate hardening viscoplastic solid $[$, i. e., the yield stress $\sim$ (effective strain-rate) ${ }^{n}$, where $n$ is the strain-rate hardening exponent (henceforth called simply as "hardening exponent"). The analysis uncovers a rich variety of regimes of hole growth some of which correspond to the experiments of Reiter [1].

The model consists of a flat film of initial thickness $h_{o}$ with a hole of radius $R_{o}$ (see FIG. 1); we focus on the growth of an existing hole rather than the mechanism of initial hole formation. As the hole grows, the radius increases to $r_{o}$ and the thickness becomes inhomogeneous. The aim of the analysis is to obtain the rate of the hole growth and the profile of the film including the height of the rim.

Capillary forces are taken to be responsible for hole growth. As the hole grows there is a net reduction in

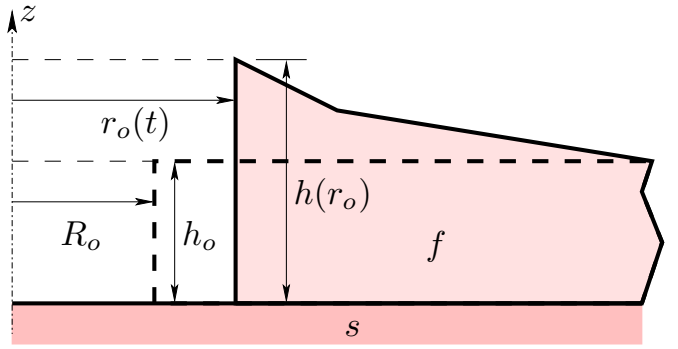

FIG. 1: Plane stress model of hole growth. The dashed configuration is the initial configuration of the film while the solid lines represent the configuration at time $t$.

interfacial energy, the rate of which is given by

$$
\dot{E}_{s}=2 \pi|S| r_{o} \dot{r}_{o}
$$

with $|S|=\left|\gamma_{s a}-\gamma_{s f}-\gamma_{f a}\right|$ is the spreading coefficient, where $\gamma_{s f}$ is the interfacial energy between the film and the substrate, $\gamma_{s a}$ is the surface energy of the substrate in contact with air and $\gamma_{f a}$ is the surface energy of the film in contact with air. As the film grows, the height $h\left(r_{o}\right)$ of the rim increases and so does the surface area of the cylindrical surface of the hole. The rate of increase in the surface energy is given by

$$
\dot{E}_{f}=2 \pi \gamma_{f a}\left(h\left(r_{o}\right)+h^{\prime}\left(r_{o}\right) r_{o}\right) \dot{r}_{o} .
$$

(We neglect the rate of increase of surface area on the top surface of the film, and this slightly overestimates the hole growth velocity). Applying principle of conservation of energy, we get

$$
\dot{E}_{s}=\dot{E}_{f}+D_{p}
$$

where $D_{p}$ is the rate of plastic dissipation in the deforming film.

The plastic dissipation $D_{p}$ (see final expression (14) below) is obtained as follows. It is assumed that the film can slip freely on the substrate, i. e., the shear stress at the film substrate interface vanishes - this assumption 
is justified by the fact that the film [1] is deposited on a monolayer of PDMS. Neglecting inertial effects, dynamics provides a relation between the stress components (a plane stress assumption with a vanishing component of stress normal to the substrate $\left(\sigma_{z z}=0\right)$ is made)

$$
\frac{\mathrm{d} \sigma_{r r}}{\mathrm{~d} r}+\frac{\sigma_{r r}-\sigma_{\theta \theta}}{r}=0
$$

where $\sigma_{r r}$ and $\sigma_{\theta \theta}$ are the radial and circumstantial components of the Cauchy stress tensor. Nonlinear kinematics of large deformation is treated; the radial coordinate of the undeformed film is described by $R$ and that of the deformed film by $r$. The deformation map can be expressed as $r=r(R, t)$ i. e., a point which was at a radial distance of $R$ at the initial instance will move to a radial position $r$ at time $t$. In particular, $r_{o}(t)=r\left(R_{o}, t\right)$ is the radius of the deformed hole. The radial velocity is the time derivative of $r$ defined as $v=\partial r(R, t) / \partial t=\dot{r}(R, t)$; the radial velocity of expansion of the hole is denoted as $\dot{r}_{o}=\dot{r}\left(R_{o}, t\right)$. The stretching rates $\epsilon_{r r}, \epsilon_{\theta \theta}$ and $\epsilon_{z z}$ are expressed in terms of the velocity field as

$$
\epsilon_{r r}=\frac{\partial v}{\partial r}, \quad \epsilon_{\theta \theta}=\frac{v}{r}, \quad \epsilon_{z z}=-\left(\epsilon_{r r}+\epsilon_{\theta \theta}\right) .
$$

Evidently, the kinematics ensures that the resulting deformation is incompressible.

We neglect elastic strains and treat the deformation as completely plastic. The polymeric material is taken to be a rate-sensitive plastic material (viscoplastic material) with power law strain rate hardening. The effective stress and the effective plastic strain rate at a material point are related by

$$
\sigma_{e}=k \varepsilon^{n}
$$

where $\sigma_{e}=\sqrt{\frac{3}{2} s_{i j} s_{i j}}$ with $s_{i j}=\sigma_{i j}-\frac{1}{3} \sigma_{k k} \delta_{i j}$ is the deviatoric stress tensor, $\varepsilon$ is the effective plastic strain rate defined as $\varepsilon=\sqrt{\frac{2}{3} \epsilon_{i j} \epsilon_{i j}}, k$ is a material constant and $n$ is the hardening exponent. The polymeric material is assumed to obey an associative flow rule[6], i. e.,

$$
\epsilon_{i j}=\lambda s_{i j}
$$

where $\lambda$ is a number; the associative rule ensures that the plastic strain rate is normal to the yield surface. Relations (4), (5), (6) and (7) provide a complete set of equations for investigating hole growth in the polymeric film.

Expression (7) can be used to express the nonvanishing stresses in terms of the stretching rates as

$$
\sigma_{r r}=\frac{2 \epsilon_{r r}+\epsilon_{\theta \theta}}{\lambda}, \quad \sigma_{r r}-\sigma_{\theta \theta}=\frac{\epsilon_{r r}-\epsilon_{\theta \theta}}{\lambda} .
$$

These expressions can be substituted in (6) to find $\frac{1}{\lambda}=$ $\frac{2}{3} k \varepsilon^{n-1}$ - the stresses are thus expressed completely in terms of the stretching rates.
On substituting the above information into the equilibrium equation (4), we get,

$$
\frac{\mathrm{d}}{\mathrm{d} r}\left(\varepsilon^{n-1}\left(2 \frac{\mathrm{d} v}{\mathrm{~d} r}+\frac{v}{r}\right)\right)+\varepsilon^{n-1} \frac{1}{r}\left(\frac{\mathrm{d} v}{\mathrm{~d} r}-\frac{v}{r}\right)=0,
$$

where

$$
\varepsilon=\sqrt{\frac{2}{3}\left[\left(\frac{\mathrm{d} v}{\mathrm{~d} r}\right)^{2}+\left(\frac{v}{r}\right)^{2}+\left(\frac{\mathrm{d} v}{\mathrm{~d} r}+\frac{v}{r}\right)^{2}\right]} .
$$

The solution of (9) provides an expression for the radial velocity

$$
v(r)=\dot{r}=\dot{r}_{o}\left(\frac{r}{r_{o}}\right)^{-\frac{1+n}{2 n}}
$$

This equation can be used to obtain an expression for the thickness profile of the film

$$
h(r, t)=h_{o}\left(\frac{r}{R}\right)^{\frac{1-n}{2 n}}, R=\left(r^{\frac{1+3 n}{2 n}}-r_{o}^{\frac{1+3 n}{2 n}}+R_{o}^{\frac{1+3 n}{2 n}}\right)^{\frac{2 n}{1+3 n}}
$$

To calculate the plastic dissipation, we substitute all the relevant quantities into the relation

$$
D_{p}=2 \pi \int_{r_{o}}^{\infty} k \varepsilon^{n+1} h(r) r \mathrm{~d} r
$$

to obtain

$$
D_{p}=2 \pi \alpha k h_{o} r_{o}^{2}\left(\frac{\dot{r_{o}}}{r_{o}}\right)^{n+1} F_{n}\left(\frac{r_{o}}{R_{o}}\right)
$$

with

$$
\begin{aligned}
\alpha & =2(3)^{-\frac{1+n}{2}}\left(1+3 n^{2}\right)^{\frac{n-1}{2}} n^{-n} \\
F_{n}(x) & ={ }_{2} F_{1}\left[\frac{1-n}{1+3 n}, \frac{1+3 n^{2}}{1+3 n} ; \frac{2+3 n+3 n^{2}}{1+3 n} ;\left(1-x^{-\frac{1+3 n}{2 n}}\right)\right],
\end{aligned}
$$

where ${ }_{2} F_{1}$ is the hypergeometric function.

Using (11), (2) and (14) in (3), the rate of hole growth is obtained as

$$
\frac{\dot{r_{o}}}{R_{o}}=\frac{1}{t_{o}}\left(1-\beta\left(\frac{r_{o}}{R_{o}}\right)^{\frac{1-3 n}{2 n}}\right)^{\frac{1}{n}}\left(F_{n}\left(\frac{r_{o}}{R_{o}}\right)\right)^{-\frac{1}{n}} \frac{r_{o}}{R_{o}}
$$

where $t_{o}$ is an intrinsic time constant of the film/substrate system defined as

$$
t_{o}=\sqrt[n]{\frac{\alpha k h_{o}}{|S|}}
$$

and

$$
\beta=\frac{1+n}{2 n} \frac{\gamma_{f a}}{|S|} \frac{h_{o}}{R_{o}}
$$


The rate of hole growth is controlled by two parameters $\beta$ which is a measure of capillary forces, and $n$ which is the strain-rate hardening exponent.

The solution to (15) can be investigated analytically in two limits, $t \ll t_{o}$ and $t \gg t_{o}$. In the short-time limit $\left(t \ll t_{o}\right)$, (15) reduces to

$$
\frac{\dot{r}_{o}}{R_{o}} \approx \frac{1}{t_{o}}(1-\beta) \frac{r_{o}}{R_{o}}
$$

since $F_{n}(1)=1$; thus the hole growth is exponential for short times. It is clear that for the hole to grow the parameter $\beta$ must be smaller than unity which provides a condition on the initial radius of the hole that allows for hole growth, i. e., $\beta<1$ implies

$$
R_{o}>R_{c}=\frac{1+n}{2 n} \frac{\gamma_{f a}}{|S|} h_{o}
$$

where $R_{c}$ is the critical radius for hole growth $(\beta=$ $R_{c} / R_{o}$ ). If the initial radius of the hole is less than $R_{c}$, the hole does not grow. As in a fluid film, the analysis for critical hole radius is most relevant when the film is sufficiently thick to ignore the excess intermolecular interactions.

The long-time behaviour $\left(t \gg t_{o}\right)$ of the film is investigated in two different regimes of $n$. First, when $n>\frac{1}{3}$, the rate of growth (15) for $t \gg t_{o}$ reduces to

$$
\frac{\dot{r_{o}}}{R_{o}} \approx \frac{L_{n}}{t_{o}} \frac{r_{o}}{R_{o}}
$$

$\left(L_{n}=\lim _{x \rightarrow \infty} F_{n}(x)^{-\frac{1}{n}}\right)$ i. e., the growth continues to be exponential but with a different exponent $L_{n} / t_{o}$. A plot of $L_{n}$ as a function of $n$ is shown in FIG. 2. Thus, although the short time exponent depends on the initial radius $R_{o}$ of the hole (through $\beta$ ), the long time behaviour is independent of the initial size of the hole. Moreover, if the critical radius is small $\left(R_{c} \ll h_{o}\right)$, then the long time growth rate of small unstable holes $(\beta \ll 1)$ slows down compared to the initial growth rate $L_{n}<(1-\beta)$.

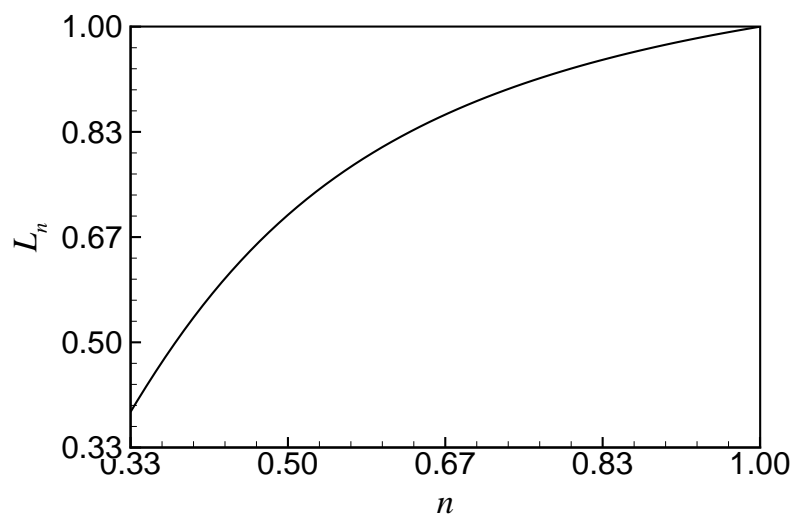

FIG. 2: Plot of $L_{n}$ vs. $n$.
When $n<\frac{1}{3}$, the long-time behaviour is very different from the above case. Indeed, the hole radius does not become indefinitely large, but attains a asymptotic value $r_{m}$ given by

$$
r_{m}=\beta^{-\frac{2 n}{1-3 n}} R_{o}
$$

A straightforward analysis shows that the approach of $r_{o}$ towards $r_{m}$ is governed by

$$
\frac{\overline{\Delta r_{o}}}{r_{m}} \approx-\frac{1}{t_{o}}\left(\frac{1-3 n}{2 n F_{n}\left(r_{m} / R_{o}\right)}\right)^{\frac{1}{n}}\left(\frac{\Delta r_{o}}{r_{m}}\right)^{\frac{1}{n}}
$$

where $\Delta r_{o}=r_{m}-r_{o}$. Thus, $r_{o}$ approaches $r_{m}$ at a power law rate governed by the reciprocal of the hardening exponent, $1 / n$.

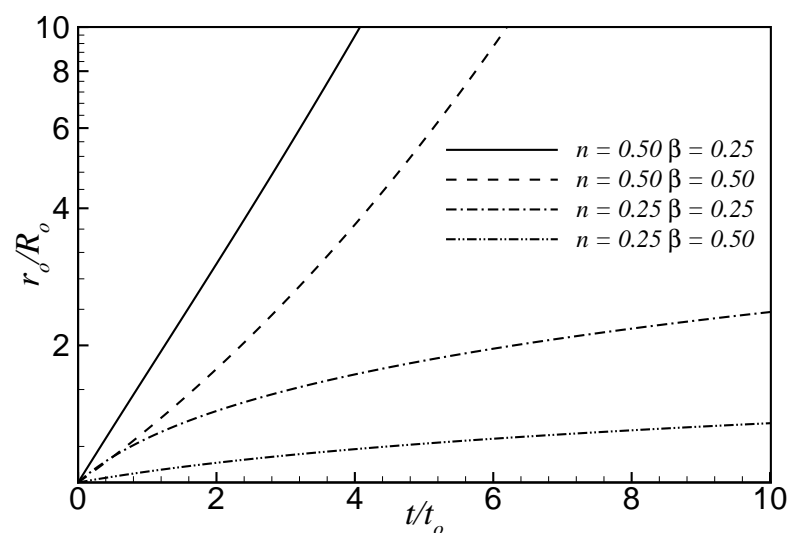

FIG. 3: Hole radius as a function of time for various combinations of $n$ and $\beta$.

The complete solution for (15) for various combinations of $n$ and $\beta$ are shown in FIG. 3. All the features noted in the asymptotic analysis are reproduced. It is clear that for a given value of $\beta$ the rate of growth is larger when $n$ is larger, and for a given $n$, the rate of growth is larger when $\beta$ is smaller.

The time evolution of the film is also of interest; for $r \gg r_{o}$, an asymptotic expression for the increase in the thickness of the film can be obtained (from (12)) as

$$
\Delta h(r, t)=\frac{(1-n)}{(1+3 n)} \frac{\left(r_{o}(t)^{\frac{1+3 n}{2 n}}-R_{o}^{\frac{1+3 n}{2 n}}\right)}{r^{\frac{1+3 n}{2 n}}}, \quad\left(r \gg r_{o}\right)
$$

FIG. A shows snapshots of film profiles for two different values of $n$ for various times. A 3D view of the film profile is included in FIG. 5 for visualistion.

Another quantity, important from the experimental point of view, is the dependence of the height of the rim on its radius. From (12), it is evident that

$$
\frac{h\left(r_{o}\right)}{h_{o}}=\left(\frac{r_{o}}{R_{o}}\right)^{\frac{1-n}{2 n}}
$$



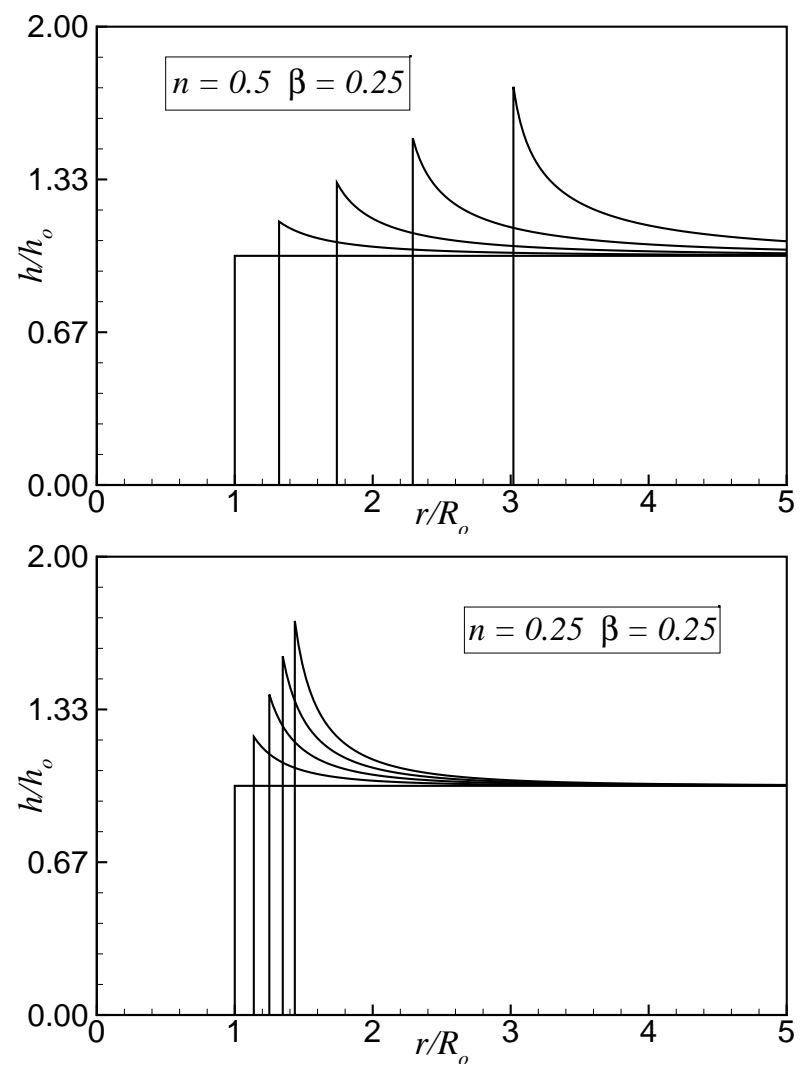

FIG. 4: Time evolution of film profiles. The profiles shown are for $t / t_{o}=0.0,0.5,1.0,1.5,2.0$.

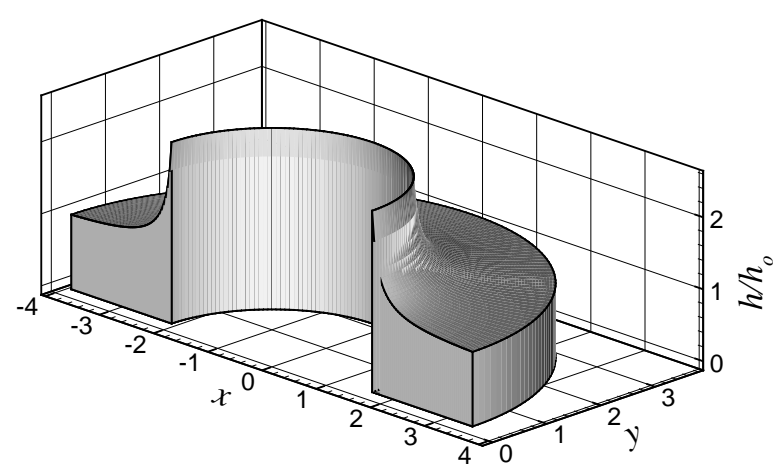

FIG. 5: 3D depiction of film profile. $n=0.25, \beta=0.25, t / t_{o}=$ 5.0 .

which is, in general, a power law. However, if $n$ is close to $1 / 3$, the exponent is approximately unity. In fact, when $n=0.25, h\left(r_{o}\right) \sim r_{o}^{1.5}$ and if $n=0.4$, then $h\left(r_{o}\right) \sim r_{o}^{0.75}$ which implies that the relationship between the height of the rim and the radius of the hole is nearly linear in this regime.

The main conclusions of this Letter are summarised as follows: (i) There is a critical value for the initial size of the hole. If the size of the hole is larger than this value, the hole grows. (ii) The short time growth velocity of the hole is exponential and depends on the initial size of the hole. (iii) The long time growth velocity of the hole depends on the strain-rate hardening exponent $n$. When $n>\frac{1}{3}$, the hole radius grows exponentially with time with a rate of growth that does not depend on the initial size of the hole. For $n<\frac{1}{3}$, the hole growth slows down and the radius of the hole attains an asymptotic value $r_{m}$ that depends on the initial size of the hole. (iv) The height of the rim is approximately linearly related to the radius of the hole when $0.25<n<0.4$.

The recent observations of Reiter[1] on hole growth in polystyrene films near glass transition temperature can be explained by the above theoretical results, supporting the premise that the hole growth in solid polymer films near $T_{g}$ is governed largely by yielding and subsequent plastic flow which provides the dominant dissipative mechanism. The theory also predicts a rich variety of growth behaviours which may help in the design and interpretation of new controlled experiments. Our theory for plastically deforming solids together with other recent theories of hole growth in viscoelastic fluids $7,8,8,9,10$ and shear thinning viscous fluids 11 provide complimentary models of dewetting over a wide range of temperatures from below $T_{g}$ to well above $T_{g}$.

Stimulating discussions with G. Reiter are gratefully acknowledged. This work was partially supported by Department of Science and Technology, and Samtel Color Ltd.

vbshenoy@iitk.ac.in ashutos@iitk.ac.in

[1] G. Reiter, Phys. Rev. Lett. 87, 186101 (2001).

[2] C. Redon, F. Brochard-Wyart, and F. Rondelez, Phys. Rev. Lett. 66, 715 (1991).

[3] A. Oron, S. H. Davis, and S. G. Bankoff, Rev. Mod. Phys. 69, 931 (1997).

[4] G. Reiter, personal communication (2002).

[5] B. Crist, in Materials Science and Technology, edited by R. W. Cahn, P. Haasen, and E. J. Kramer (VCH Publishers Inc., New York, 1993), vol. 12, pp. 427-469.

[6] A. S. Khan and S. Huang, Continuum Theory of Plasticity (John Wiley \& Sons, Inc., New York, 1995).

[7] M. A. Sapid and G. M. Homsy, Phys. Fluids 8, 460 (1996).

[8] F. Brochard-Wyart, G. Debergeas, R. Fondecave, and P. Martin, Macromolecules 30, 1211 (1997).

[9] K. Jacobs, R. Seemann, G. Schatz, and S. Herminghaus, Langmuir 14, 4961 (1998).

[10] S. Herminghaus, R. Seemann, and K. Jacobs, arXiv:cond-mat/0201193.

[11] F. Saulnier, E. Raphaël, and P.-G. de Gennes, arXiv:cond-mat/0201528. 